\title{
Lessons learned from COVID-19 outbreaks profile in combination with pathology, diagnosis, treatment and vaccines
}

\author{
Ruizhe Liu ${ }^{1, a, *, \dagger}$, Enqi Zhang ${ }^{2, \text { b }, *, \dagger}$ \\ ${ }^{1}$ Dalian No.24 high school, 116001, China \\ ${ }^{2}$ EMcGill University, Faculty of Science, H3A 0C6, Montreal, Canada \\ †These authors contributed equally to this paper.
}

\begin{abstract}
COVID-19 is an infectious disease caused by the SARS-CoV-2 virus, which may lead to severe respiratory infection. Since December 2019, the first COVID-19 case was detected in Wuhan, Hubei Province, China. The virus has been spread worldwide, and many countries are still struggling with disease control. Therefore, this revision would provide experience accumulated and the lessons learned by China in the pandemic. The current work reviewed the basic information about COVID-19 in the aspects of pathology, diagnosis, treatment, prevention, and the major pandemic outbreaks in China. By summarizing and analyzing the measurements taken by the Chinese government and their corresponding outcomes on public health, it determined the progress made on the policies and their efficiency in the control of pandemics. With the sharing of these lessons, other countries can learn from establishing the COVID-19 surveillance system in China that would support their struggle on domestic public health.
\end{abstract}

\section{Introduction}

On December 31, 2019, cases of pneumonia detected in Wuhan, China, are first reported to the World Health Organization (WHO), later attributed to a coronavirus, now named SARS-CoV-2. By January 30, 2020, WHO determined the virus to be a public health emergency of international concern (PHEIC). Since then, the pandemic was well underway, and the virus has spread to every continent except Antarctica.

In China, the government undertook drastic measures against the coronavirus. A lockdown was imposed on Wuhan, aggressively testing and forcibly rounding up potential carriers in makeshift quarantine centers. Then, the containment was extended to most of the country. By the time those interventions began. China has successfully contained its spread through public health measures, for instance, testing, contact tracing, and isolation of confirmed and suspected cases. Besides China, more than one hundred countries implemented either a full or a partial shutdown, required mask-wearing, and enforced physical distancing to control the spread of the virus and reduce pressure on their health systems. Although these measures slowed the pace of infection, many nations' societal and economic consequences have been grim. Because of a mixture of partial and mishandled responses, a lack of cooperation and coordination across nations, and a failure of compliance with established norms and international agreements, the COVID19 pandemic has led to a dramatic loss of human life worldwide. It presents an unprecedented challenge to public health, food systems, and the world of work. Tens of millions of people are at risk of falling into extreme poverty, and by the end of 2020, the number of undernourished people estimated at nearly 132 million [1].

In this paper, the information such as virus and pathology, diagnosis, treating options, and vaccines on COVID-19 was reviewed; the epidemic outbreak in Wuhan, Beijing, Dalian, China was revised; lessons learned from this pandemic and perspective for referential indication were summarized.

\section{The COVID-19}

\subsection{Overview}

Coronavirus is a large family of viruses that causes an infection in one's nose, sinuses, or upper throat. The illness caused by coronavirus can range from the common cold to more severe diseases, such as Middle East Respiratory Syndrome (MERS-CoV) and Severe Acute Respiratory Syndrome (SARS-CoV). A novel coronavirus (nCov) was identified in 2019 in Wuhan, China, that had not been identified in humans previously, named '2019-nCoV' or 'COVID-19' by the World Health Organization (WHO), oversees the current outbreak of pneumonia [1-2]. The COVID-19 pandemic had affected lives across the world. Many countries had to period lockdowns resulted in severe economic consequences. Many countries' embassies period stopped to renew or issue new visa caused international

*Corresponding author Email:aliuruizhe2022@outlook.com, benqi.zhang@mail.mcgill.ca. 
travel to become extremely difficult. On the one hand, many companies had to start to work from home, students started to study online, and people ordered their groceries online. On the other hand, others have no choice but to be exposed to the virus with very little protection (wearing facemasks) to keeping society functioning. Our status as individuals and as members of society determine the pandemic's different impact and its consequences.

\subsection{Virus and Pathology}

COVID-19 is a pathogenic virus. The virus causing it is SARS-CoV-2. The complete genome of SARS-CoV-2 from Wuhan, China, was published on January 17, 2020, on the National Center for Biotechnology [3]. The genome of SARS-CoV-2 is a 29,903 bp single-stranded RNA (ss-RNA) coronavirus. Coronaviruses are zoonotic, which means they can transmit between animals and humans [4]. The source of the SARS-CoV-2 is to be determined, but investigations are ongoing to identify the zoonotic source of the outbreak. However, three significant work areas are already ongoing in China to advise our awareness of the outbreak's pathogenic origin. These include 1) environmental sampling from the Huanan Wholesale Seafood Market as well as other area markets, 2) early investigations of cases with symptoms occurring near/in Wuhan during December 2019, and 3) the collection of information of the origin and type of wildlife species marketed on the Huanan market and the destination of those animals after the market has been closed [5-8].

SARS-CoV-2 entered into cells caused the release of a significant amount of pro-inflammatory cytokines, which aggravate pneumonia and acute respiratory distress syndrome (ARDS). The reasons for its high morbidity and mortality are yet to be revealed. Autopsy findings are crucial to gaining a better understanding of the pathobiology of disease. In February 2020, Xu et al. [9] conducted core biopsies of a man who experienced 14 days of progressive respiratory symptoms and sampled his lung, heart, and liver. Another 6 teams conducted minimally invasive autopsies, and 21 teams conducted complete autopsies in 341 patients who died because of COVID-19 individually [10-26]. The results show that the lung's histological feature diffuses alveolar damage with hyaline membrane formation, along with microthrombi in small pulmonary vessels. However, more studies are needed to describe the extrapulmonary manifestations of the disease entirely.

\subsection{Diagnosis}

Up to 18:51 GMT, April 08 2021, COVID-19 infected patients confirmed in the world is $134,190,375$, with 2,907,652 death and 108,123,067 recovered. According to the WHO [27], the most common symptoms of COVID-19 are fever, dry cough, and fatigue, and some common symptoms are shortness of breath, loss of appetite, confusion, persistent pain or pressure in the chest, and temperature higher than $38^{\circ} \mathrm{C}$. SARS-CoV-2 infect- ed people of all ages. However, the WHO [27] published that two groups of people are at a higher risk of getting severe COVID-19 disease: older than 60 years or have health conditions like diabetes, cardiovascular disease, chronic respiratory disease, cancer, hypertension, or chronic liver disease.

So far, the available diagnosis tools have been based on a) viral gene detection, b) human antibody detection, and c) viral antigen detection. Nucleic acid amplification testing (NAAT) to detect viral genes is the preferred initial diagnostic test for COVID-19 [28]. In general, the detection of the viral has been almost solely dependent on NAAT. The human antibody detection test was beneficial with vast immune screening, but it did not confirm the virus's presence. The antigen test is still under development.

\subsection{Treating options}

Most people infected with COVID-19 would be able to recover without have been hospitalized. Getting enough rest, staying well hydrated, and taking medication to release fever, aches, and pains would help with COVID19. For non-hospitalized adults and children over age 12 with mild to moderate COVID-19 symptoms at risk for developing severe COVID-19 or being hospitalized for it, the U.S. Food \& Drug Administration (FDA) granted emergency use authorization to two monoclonal antibody treatments, bamlanivimab, and casirivimab and imdevimab combination. Beyond that, the FDA had also authorized the antiviral drug, remdesivir, baricitinib combined with remdesivir, and anticoagulation drugs used for people who had been hospitalized with COVID19. During hospitalization, the health care team would keep an eye on the symptoms getting worse or need super concern. If a patient was starting to require higher levels of blood pressure support or their respiratory situation was getting compromised, they would be admitted to an intensive care unit (ICU). A ventilator or less invasive oxygen therapies, such as high-flow nasal cannulas, could treat some COVID-19 patients.

\subsection{Vaccines}

Up to February 08, 2021, 8 brands of COVID-19 vaccine had been authorized worldwide to the public. These vaccines could be categorized into five types: mRNA vaccine, adenovirus-based vaccine, inactivated SARSCoV-2 virus vaccine, protein-based vaccine, and viral vector vaccine. The mRNA vaccine was coded for the virus's spike protein and condensed in a lipid nanoparticle. Once injected, the cells spitted out the spike protein, triggered the body's immune system to recognize the virus. Both Pfizer-BioNTech and Moderna were mRNAtype vaccines. Their efficacies were about $95 \%$, and $100 \%$ preventing hospitalization and death. The PfizerBioNTech vaccine required storage at about $-70{ }^{\circ} \mathrm{C}$, which required specialized freezers. However, the Moderna vaccine could stable at 2 to $8{ }^{\circ} \mathrm{C}$ for up to 30 days and be stored for up to six months at $-20^{\circ} \mathrm{C}$. AstraZeneca-University of Oxford, Johnson \& Johnson, and 
Russia's Sputnik V Vaccine were adenovirus-based vaccines. Overall efficacy of AstraZeneca-University of Oxford was about $70 \%$. Johnson \& Johnson demonstrated $66 \%$ efficacy in the global clinical trial and was slightly higher in the U.S, which was $72 \%$. Russia's Sputnik V Vaccine claimed its efficacy as high as $91.4 \%$. China-based Sinovac Biotech developed inactivated SARS-CoV-2 virus vaccine. It demonstrated noticeably varying efficacy rates from $50.38 \%$ to $91.25 \%$ depends on the clinical trials. Novavax announced a proteinbased vaccine and hit the primary endpoint with an efficacy of $89.3 \%$ in its Phase III trial in the UK. Last but not least, Cansino Biologics co-developed a viral vector vaccine with the Chinese military. It loaded an antigen from the SARS-CoV-2 virus onto an adenovirus. It could prevent $65.7 \%$ symptomatic cases and $90.98 \%$ efficacy in severe disease.

According to the New York Times [29], up to April 8,2021 , more than 710 million vaccine doses had been dispensed worldwide, equal to 9.2 doses for every 100 people. Mainland China has administered 149,071,000, equal to 11 does for every 100 people. China was already way ahead of the average world level in vaccine injection rate.

This COVID-19 pandemic has caused a worldwide sudden. In this paper, the whole pandemic has been chronological reviewed.

\section{Epidemic revision}

\subsection{Pandemic in Wuhan}

\subsubsection{Timeline}

On December 30, 2019, several "viral pneumonia" cases with an unknown origin were notified and reported in Wuhan, Hubei Province in China. Subsequently, on 9 January 2020, the Chinese Center for Disease Control and Prevention identified this outbreak was caused by a novel coronavirus, which has later been named severe acute respiratory syndrome coronavirus 2(SARS-CoV-2) [32]. WHO called the global alert by the end of January 2020, and China had 7736 confirmed cases with 170 deaths meanwhile [32]. Within one month, imported cases outside China emerged and arose worldwide concerns. The outbreak in Wuhan has transformed into a global pandemic that continues to this day.

\subsubsection{Originality}

The origin of the COVID-19 in the outbreak in Wuhan is controversial and remains unclear because there is no conclusive evidence to confirm the exact origin of the virus. However, relative scientific research has shown that SARS-CoV-2 might be transmitted from bats through unknown intermediate to humans. SARS-CoV-2 is a beta coronavirus that can infect mammals and potentially cause severe respiratory tract infections [30]. According to the virus-genome sequencing result and evolutionary analysis, the bat is the most likely natural host of virus origin [30]. Besides, the Seafood Market in Wuhan also helped spread the virus, and many have suspected the live animals sold there might be contaminated by the virus; however, it is not where the outbreak first emerged [30,31]. The Figure 1 below from demonstrates the transmission pathway of COVID-19 in Wuhan outbreak [32].

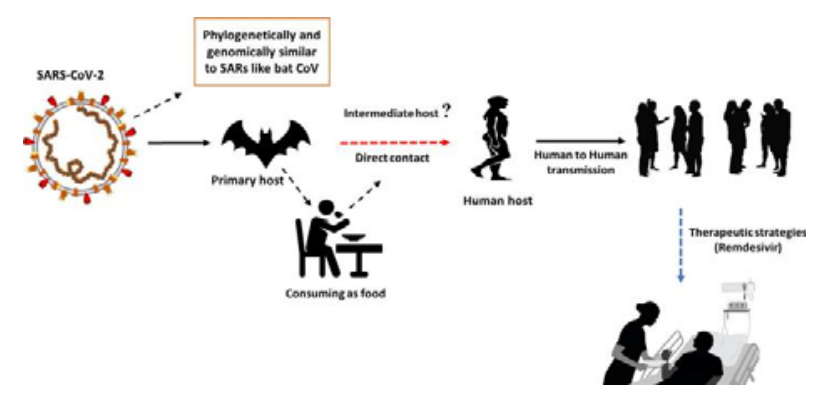

Fig.1 Directly consumed or having contact with the infected wild animals would lead to animal to human transmission.

\subsubsection{New characteristics}

The outbreak in Wuhan is the first time that SARSCoV-2 came into public sight. Therefore, the investigations around the pathogens, transmission mechanisms, the syndromes developed, and the prevention measures were just started, and local government was not that experienced. As mentioned in 2.1.2, the epidemic outbreak most likely originated from bats and spreaders out through the Seafood Market [31]. While later, the emerged population of patients who have never been to the market indicated the human-to-human transmission is possible [30].

\subsubsection{Regulation and handling strategies}

Domestically, the Chinese government had imposed strict lockdown to Wuhan and Hubei Province after the outbreak. The other provinces were also enforced to traffic restriction, house quarantine, social distancing, and the report of suspected and confirmed cases [33]. The strong adherence to these strategies of people positively affected the control of the epidemic in this outbreak [34]. Moreover, the mobilization of clinical resources to Wuhan and the build-up of mobile cabin hospitals also helped stop the spread of the virus. Internationally, flights to China had been canceled, and custom quarantine followed by self-isolation for 14 days have been applied.

\subsection{Pandemic in Beijing}

\subsubsection{Timeline}

The second outbreak of COVID-19 happened in Beijing. China has brought public concern and the focus on the routine of imported cases. On 11 June 2020, a 52-year 
old man was diagnosed as COVID-19 in Beijing, while he has no known exposure to any COVID-19 confirmed cases [39]. After 56-day zero new case interval, the resurgence of the COVID-19 case indicated the potential transmission to others might have already occurred [39]. Soon on 12 June 2020, close contacts and environmental samples located and collected through contact tracing were sent to complete the qRT-PCR test $[38,39]$. As a result, all close contact samples were negative, while two environmental samples from Xinfadi Market (XFDM) were positive [39]. To investigate the potential of the environment to human transmission, more samples were collected from 10 million citizens, and 5342 environmental samples were checked by 10 July 2020 [39]. Finally, the positive results among these samples came from people with experience of working, visiting, or having contact with the employees or visitors in this market.

\subsubsection{Originality}

Based on 2.2.1, the test results combined with the contact tracing and exposure history point out that the outbreak in Beijing is single-originated [39]. Xinfadi Market is the only resource of the virus. To determine the specific origin of the virus, further research on the spatial distribution of employees with positive test results has been done. The outcome indicated the basement of XFDM had the highest spatial clusters, especially in the seafood section $[37,38,39]$. By antibody test, it was confirmed that the virus had been introduced into Beijing before June. However, the comparison between the genome sequences from the SARS-CoV-2 detected XFDM found in previous outbreaks illustrated that the virus strain found in Beijing did not come from any strain previously circulating in China they were distinct [39]. Thus this virus strain was likely to be imported. Consequently, the detailed investigation in XFDM showed that the environment likely caused this COVID-19 in Beijing to human transmission coming from imported food carrying coronavirus via cold-food chain.

\subsubsection{New characteristics}

Firstly, the ancestral strain detected on salmon in XFDM in this outbreak was not indigenous to China. Instead, it was mainly identified in Europe [39]. Secondly, even though human-to-human transmission has played a major role in increasing COVID-19 cases, environment-tohuman transmission was also essential in this event. Therefore, the outbreak in Beijing was the first epidemic caused by environment-to-human transmission from the imported cold-food chain in China.

\subsubsection{Regulation and handling strategies}

To prevent the reintroduction of contaminated food and the initiation of another outbreak via environment to human transmission, China had applied border control and quarantine and checked and traced the cold-food chain in XFDM to ensure it would not cause further transmission [39]. More strict disinfection and examination of the goods imported were enforced as well.

\subsection{Pandemic in Dalian}

\subsubsection{Timeline}

The resurgence of COVID-19 in Dalian, Liaoning Province in China from July to August 2020 started on $9 \mathrm{Ju}-$ ly, when the first patient had developed the syndrome. While the first case notified and reported by the local hospital was on 22 July. And the local Disease Control Center and government respond quickly to carry out the large-scale nucleic acid test to the whole city, especially in the communities around Dalianwan Street [35]. 118 cases had been detected, and $95.76 \%$ of cases were identified through contact tracing and investigation in communities.

\subsubsection{Originality}

Like the outbreak in XFDM, this epidemic originated from a cold-seafood chain company and was presumed to be initiated by the environment-to humantransmission. The contaminated seafood on the chain transmitted the virus to the employees and caused further human-to-human transmissions among workers.

\subsubsection{New characteristics and Strategies}

This outbreak is still an example of imported virus strain, while the diagnosed cases and close contacts are lesser than the number in the Beijing XFDM outbreak. The decrease in the number of patients and the early detection helped control the spread of the virus. After the first case identified on 22 July 2020, Dalian city quickly and decisively blocked the transmission chain, isolated the staff and family members of a food company who had the case at the first time, extensively carried out nucleic acid testing for all staff, defined close contacts. It then expanded the scope of screening and control of close contacts [35]. These measures successfully minimized the influence and the magnitude of the epidemic and contributed the better public health.

\subsection{Summary, lessons learned, and referential indications}

Through this overview of three major outbreaks of COVID-19 in China, China has accumulated more experience in detecting infection, clinical care, and the quarantine and self-isolation in both communities and borders to prevent transmission. The government's preparation for the outbreak is more sufficient, which can be proved by the reduced number of identified cases in the Dalian Outbreak. In Wuhan, China learned to impose lockdown to restrict the spread of the virus over provinces and some practice in treating COVID-19 infection. Later in Beijing, the unexpected virus resource gives alert, so the border control, isolation, and contact tracing 
in communities have been enhanced. Even though the outbreak caused by cold-chain contaminated food resurged in Dalian, the response of the government and disease control center made the control of the epidemic more effective.

\section{Conclusion}

This paper presents the basic information of COVID-19 and its three main outbreaks in China. We have discussed the pathogenic mechanism, the diagnosis, treatment, and prevention in the first part, followed by the detailed review of three outbreaks that happened in $\mathrm{Wu}-$ han, Beijing, and Dalian. Through this overview and analysis, we have identified the diagnostic technique, the pathology, and the vaccine developed and used of COVID-19. We have also reviewed and summarized the progression made by the Chinese government on the control of the transmission of the virus to maintain public health and the lessons it learned from the past outbreaks. Moreover, the multiple pathways for virus transmission, the rise of a new variation of strain, and many infected populations are all difficulties left for the public health system to solve. The information provided in this essay would give a review for the experience accumulated specifically in China in the past two years since the first outbreak in Wuhan. Further international exchange of experience in pandemic control and cooperation among nations are expected.

\section{References}

1. Corman VM et al (2020) Detection of 2019 novel coronavirus (2019-nCoV) by real-time RT-PCR. Eurosurveillance 25(3)

2. Fong SJ, Li G, Dey N, Crespo RG, Herrera-Viedma E (2020) composite monte carlo decision making under high uncertainty of novel coronavirus epidemic using hybridized deep learning and fuzzy rule induction. arXiv preprint arXiv:2003.09868

3. Wuhan seafood market pneumonia virus isolate Wuhan-Hu-1, complete genome. Nucleotide, National Center for Biotechnology Information (NCBI), National Library of Medicine (US), National Center for Biotechnology Information, Bethesda, MD, https://www-ncbi-nlm-nih-gov.libe2.lib.ttu.edu/nuccore/1798174254 (accessed on 2020-02-28).

4. Kishor, R. S., \& Ramhari, B. M. (2020). Introduction to covid-19. Research Journal of Science and Technology, 12(4), 338-345. doi:http://dx.doi.org.lib-e2.lib.ttu.edu/10.5958/23492988.2020.00051.0

5. China just banned the trade and consumption of wild animals. Experts think the coronavirus jumped from live animals to people at a market. https://www.businessinsider.in/science/news/ch ina-just-banned-the-trade-and-consumption-of-wildanimals-experts-think-the-coronavirus-jumped- from-live-animals-to-people-at-a-market/articleshow/74309474.cms

6. Calls for global ban on wild animal markets amid coronavirus outbreak https://www.theguardian.com/science/2020/ja n/24/calls-for-global-ban-wild-animal-marketsamid-coronavirus-outbreak

7. Coronavirus closures reveal vast scale of China's secretive wildlife farm https://www.theguardian.com/environment/20 20/feb/25/coronavirus-closures-reveal-vast-scale-ofchinas-secretive-wildlife-farm-industry

8. Chinese citizens push to abolish wildlife trade as coronavirus

persists https:/www.nationalgeographic.com/animals/2 020/01/china-bans-wildlife-trade-after-coronavirusoutbreak/

9. $\mathrm{Xu} \mathrm{Z}$, Shi L, Wang Y, et al. Pathological findings of COVID-19 associated with acute respiratory distress syndrome. Lancet Respir Med. 2020;8:420422. doi: 10.1016/S2213-2600(20)30076-X.

10. Zhang H, Zhou P, Wei Y, Yue H, Wang Y, Hu M, et al. Histopathologic changes and SARS-CoV-2 immunostaining in the lung of a patient with COVID-19. Ann Intern Med. 2020;172:629-632. doi: 10.7326/M20-0533.

11. Dolhnikoff M, Duarte-Neto AN, de Almeida Monteiro RA, et al. Pathological evidence of pulmonary thrombotic phenomena in severe COVID-19. J Thromb Haemost. 2020;18:1517-1519. doi: $10.1111 /$ jth. 14844 .

12. Li G, Fox SE, Summa B, et al. Multiscale 3dimensional pathology findings of COVID-19 diseased lung using high-resolution cleared tissue microscopy. $\quad$ bioRxiv. 2020; 10.1101/2020.04.11.037473.

13. Yao XH, Li TY, He ZC, et al. A pathological report of three COVID-19 cases by minimal invasive autopsies. Zhonghua Bing Li Xue Za Zhi. 2020;49:411-417.

14. Barton LM, Duval EJ, Stroberg E, Ghosh S, Mukhopadhyay S. COVID-19 autopsies, Oklahoma, USA [published correction appears in Am J Clin Pathol. 20205;153:852] Am J Clin Pathol. 2020;153:725-733. doi: 10.1093/ajcp/aqaa062.

15. Grimes Z, Bryce C, Sordillo EM, et al. Fatal pulmonary thromboembolism in SARS-CoV-2infection. Cardiovasc Pathol. 2020;48:107227. doi: 10.1016/j.carpath.2020.107227.

16. Varga Z, Flammer AJ, Steiger P, et al. Endothelial cell infection and endotheliitis in COVID-19. Lancet. 2020;395:1417-1418. doi: 10.1016/S01406736(20)30937-5.

17. Bradley BT, Maioli H, Johnston R, et al. Histopathology and ultrastructural findings of fatal COVID-19 infections in Washington State: a case series [published correction appears in Lancet. 
2020;396:312] Lancet. 2020;396:320-332. doi: 10.1016/S0140-6736(20)31305-2.

18. Paniz-Mondolfi A, Bryce C, Grimes Z, et al. Central nervous system involvement by severe acute respiratory syndrome coronavirus-2 (SARS-CoV-2) J Med Virol. 2020;92:699-702. doi: 10.1002/jmv.25915.

19. Lacy JM, Brooks EG, Akers J, et al. COVID-19: Postmortem diagnostic and biosafety considerations. Am J Forensic Med Pathol. 2020 doi: 10.1097/PAF.0000000000000567.

20. Konopka KE, Wilson A, Myers JL. Postmortem lung findings in a patient with asthma and coronavirus disease 2019. Chest. 2020;158:E99-101. doi: 10.1016/j.chest.2020.04.032.

21. Prilutskiy A, Kritselis M, Shevtsov A, Yambayev I, Vadlamudi C, Zhao Q, et al. SARSCoV-2 infection associated hemophagocyticlymphohistiocytosis: an autopsy series with clinical and laboratory correlation. medRxiv. 2020. 10.1101/2020.05.07.20094888

22. Menter T, Haslbauer JD, Nienhold R, et al. Postmortem examination of COVID-19 patients reveals diffuse alveolar damage with severe capillary congestion and variegated findings in lungs and other organs suggesting vascular dysfunction. Histopathology. 2020 doi: 10.1111/his.14134.

23. Remmelink M, De Mendoca R, D'Haene N, De Clercq S, Verocq C, Lebrun L, et al. Unspecific post-mortem findings despite multiorgan 1 viral spread in COVID-19 patients. medRxiv. 2020. 10.1101/2020.05.27.20114363.

24. Wichmann D, Sperhake JP, Lütgehetmann M, et al. autopsy findings and venous thromboembolism in patients with COVID-19. Ann Intern Med. 2020 doi: 10.7326/M20-2003.

25. Fox SE, Akmatbekov A, Harbert JL, Li G, Quincy Brown J, Vander Heide RS. Pulmonary and cardiac pathology in African American patients with COVID-19: an autopsy series from New Orleans. Lancet Respir Med. 2020;8:681-686. doi: 10.1016/S2213-2600(20)30243-5.

26. Carsana L, Sonzogni A, Nasr A, et al. Pulmonary post-mortem findings in a series of COVID-19 cases from northern Italy: a two-centre descriptive study. Lancet Infect Dis. 2020 doi: 10.1016/S14733099(20)30434-5.

27. Coronavirus update (live): Cases and deaths from COVID-19 virus pandemic

28. Worldometers (2021).

29. World Health Organization, Coronavirus disease (COVID-19). 2020.

30. The New York Times, Tracking Coronavirus Vaccinations Around the World. 2021.

31. Guo, Y., Cao, Q., Hong, Z., Tan, Y., Chen, S., Jin, H., ... Yan, Y. (2020, March 13). The origin, transmission and CLINICAL therapies ON CORONAVIRUS disease 2019 (COVID-19) OUTBREAK - an update on the status. Retrieved April 23, 2021, from

https://mmrjournal.biomedcentral.com/articles/10.1 186/s40779-020-00240-0

32. Shereen, M., Khan, S., Kazmi, A., Bashir, N., \& Siddique, R. (2020, March 16). COVID-19 infection: Emergence, transmission, and characteristics of Human coronaviruses. Retrieved April 23, 2021, from

https://www.sciencedirect.com/science/article/pii/S2 $\underline{090123220300540}$

33. Zhang, J., Wang, X., Jia, X., Li, J., Hu, K., Chen, G., . . D Dong, W. (2020, April 15). Risk factors for Disease Severity, Unimprovement, and mortality IN COVID-19 patients IN WUHAN, CHINA. Retrieved April 23, 2021, from https://www.sciencedirect.com/science/article/abs/pi i/S1198743X20302172

34. Guan, W., Al., E., For the China Medical Treatment Expert Group for Covid-19*, Author AffiliationsFrom the State Key Laboratory of Respiratory Disease, Others, A., Others, M., \& D. B. Cines and J. B. Bussel. (2020, May 07). Clinical characteristics of Coronavirus DISEASE 2019 in China: Nejm. Retrieved April 23, 2021, from https://www.nejm.org/doi/10.1056/NEJMoa200203 $\underline{2}$

35. Zhong, B., Luo, W., Li, H., Zhang, Q., Liu, X., Li, W., \& Li, Y. (2020, March 15). Knowledge, attitudes, and practices towards COVID-19 among Chinese residents during the rapid Rise period of the Covid-19 Outbreak: A quick online cross-sectional survey. Retrieved April 23, 2021, from https://www.ncbi.nlm.nih.gov/pmc/articles/PMC709 $\underline{8034 /}$

36. Yucun, C., Shihong, Y., Qingyu, A., Liyang, P., Xiaoli, W., Jin, H., \& Dezheng, W. (2021, March 10). Epidemiological investigation of an outbreak of CORONAVIRUS disease 2019 in Dalian, Liaoning, July-August, 2020. Retrieved April 23, 2021, from http://www.jbjc.org/article/doi/10.3784/jbjc.202009 030308?pageType $=$ en

37. Zunyou Wu, M. (2020, April 07). The coronavirus DISEASE 2019 (COVID-19) outbreak in Chinasummary of a CHINA CDC Report. Retrieved April 23, 2021, from https://jamanetwork.com/journals/jama/articleabstract $/ 2762130$

38. Tian, S., Hu, N., Lou, J., Chen, K., Kang, X., Xiang, Z., . . Z Zhang, J. (2020, February 27). Characteristics of COVID-19 infection in Beijing. Retrieved April 23, 2021, from https:/www.sciencedirect.com/science/article/abs/pi i/S0163445320301018

39. Zunyou Wu, M. (2020, October 13). Time course of a Second outbreak of COVID-19 in Beijing, CHINA, June-july 2020. Retrieved April 23, 2021, from https://jamanetwork.com/journals/jama/article$\underline{\text { abstract } / 2769930}$ 